\title{
Biological Safety Evaluation of KiOmedine® CM-chitosan, an Innovative Non-animal Carboxymethyl-Chitosan Biomaterial Intended for Injectable Biomedical Applications
}

\author{
Pierre Douette $^{1}$, Mickael Chausson ${ }^{1}$, Emilie Theatre ${ }^{1}$, Catherine Philippart ${ }^{1}$, Sandrine Gautier ${ }^{1}$, \\ Jacques Bentin ${ }^{2}$, Laurence Hermitte ${ }^{1, *}$ \\ ${ }^{1}$ Kiomed Pharma, Herstal, Belgium \\ ${ }^{2}$ Department of rheumatology, Centre Hospitalier Universitaire Brugmann, Brussels, Belgium
}

Email address:

Laurence.hermitte@kiomedpharma.com (L. Hermitte)

*Corresponding author

\section{To cite this article:}

Pierre Douette, Mickael Chausson, Emilie Theatre, Catherine Philippart, Sandrine Gautier, Jacques Bentin, Laurence Hermitte. Biological Safety Evaluation of KiOmedine ${ }^{\circledR}$ CM-chitosan, an Innovative non-animal Carboxymethyl-Chitosan Biomaterial Intended for Injectable Biomedical Applications. Journal of Biomaterials. Vol. 4, No. 2, 2020, pp. 39-50. doi: 10.11648/j.jb.20200402.12

Received: September 7, 2020; Accepted: September 19, 2020; Published: September 25, 2020

\begin{abstract}
When designing innovative biomaterials, biocompatibility is regarded as a prerequisite for safe clinical use in humans. In this study, the biological safety of KiOmedine ${ }^{\circledR}$ CM-chitosan, which is a non-animal carboxymethyl chitosan biomaterial, was evaluated using a large panel of both in vitro and in vivo biocompatibility tests in accordance with the ISO 10993 series. KiOmedine ${ }^{\circledR}$ CM-chitosan was non-cytotoxic and non-genotoxic in vitro. The biomaterial was neither found to be haemolytic nor was it able to potentiate the activation of the central complement component C5a or the inflammatory mediators IL-1 $\beta$ and IL-8 in the presence of human whole blood. Furthermore, no evidence of any significant irritation, sensitization, pyrogenicity, and organ toxicity was detected in specific animal studies conducted with KiOmedine ${ }^{\circledR}$ CM-chitosan, and only minimal local tissue effects were observed after the intra-articular or intra-dermal injection of KiOmedine ${ }^{\circledR} \mathrm{CM}$-chitosan in the rabbit model. KiOmedine ${ }^{\circledR}$ CM-chitosan had minimal potential to induce immunotoxic reactions in the mouse air pouch model. Its biodegradation process was appropriately characterized at the histological level. In summary, our study represents an unprecedented body of work supporting the biological safety evaluation of KiOmedine ${ }^{\circledR}$ CM-chitosan, allowing its use in injectable medical devices.
\end{abstract}

Keywords: Carboxymethyl Chitosan Biomaterial, Biocompatibility, Toxicology

\section{Introduction}

Over the last two decades, chitosan and its chemical derivatives has become a biomaterial of growing interest in regenerative medicine research and biomedical applications $[1,2]$. Chitosan is a linear polysaccharide that consists of $\mathrm{N}$-acetyl-D-glucosamine (GlcNAc) and D-glucosamine units $(\mathrm{GlcN})$ linked by $\beta(1-4)$-bonds. Chitosan is obtained by deacetylation of chitin, a naturally occurring carbohydrate polymer that is widely distributed in nature in crustacean shells, insects, and mollusks, as well as fungal cell walls. The degree of acetylation (DA) is known to influence the solubility of chitosan and its rheological properties. Whereas chitin is considered to have DA $>90 \%$, chitosan has typically low DA $<35-40 \%[3,4]$. As a consequence, from their chemical structure, chitin and chitosan have a limited solubility in physiological aqueous systems.

In order to achieve physiological solubility, one approach consists in grafting chemical substituents onto these polymers. Carboxymethylation is one kind of carboxylation chemistry that is aimed at grafting carboxylic groups onto chitin or chitosan in the presence of monochloroacetic acid (MCA). The method for the preparation of carboxymethyl (CM) derivatives of chitin was first described in the 1960s [5]. The stereochemistry of CM derivatives is directly influenced by the chemical nature of the starting material. The Substitution of alcoholic function of the glucosamine backbone is primarily achieved with high DA chitin while 
mixed amine/alcohol reactivity is obtained with low DA chitosan [6]. Process conditions driving the substitution of the polymer are well characterized [7-10].

Biocompatibility and biodegradability are regarded as key attributes when designing innovative biomaterials for biomedical applications [1, 2]. Development efforts of chitosan-based biomaterials has so far resulted in relatively few commercial products. Difficulties in terms of developing safe and effective chitosan-based biomaterials could reside in the lack of in-depth exploration of its biocompatibility, its biodegradability, and the resulting biological response to the biomaterial in host tissues [11]. The immunoreactivity of chitosan, as well as the effect of chitosan impurities, such as bacterial endotoxins [12] or process residues [13], on the biological reactivity of commercially-available chitosan is also evidenced as a limitation in the literature.

The objective of this study was to establish the biological safety of an innovative non-animal CM-chitosan biomaterial called $\mathrm{KiOmedine}{ }^{\circledR} \mathrm{CM}$-chitosan, which is intended for injectable medical devices. The KiOmedine ${ }^{\circledR}$ biomaterial evaluated in this study is composed of a non-animal CM-chitosan that is extracted from the edible mushroom Agaricus bisporus. this report describes the results from extensive biocompatibility testing in order to support the biological evaluation of $\mathrm{KiOmedine}\left({ }^{\circledR}\right.$ as a prerequisite for its safe clinical use in humans. The biocompatibility testing plan has been designed in accordance with the ISO 10993 series defining the pre-clinical requirements for the biological evaluation of medical devices. Relevant products that are composed of hyaluronic acid (HA) were used as commercial references in implantation studies.

\section{Materials and Methods}

\subsection{Ethical Considerations}

All biocompatibility studies were conducted on sterile final biomaterial following extensive characterization and toxicological evaluation in compliance with ISO 10993-1 (2018): Biological evaluation of medical devices, Part 1: Evaluation and testing within a risk management process. All animal studies described in this study were performed in full compliance with the OECD Good Laboratory Practice (C (97) 186 Final), the FDA GLP (21 CFR 58), the ARRIVE (Animal Research: Reporting of In Vivo Experiments) guidelines, and the ISO 10993-2 (2006) for animal welfare, following prior approval by the Institutional Animal Care and Use Committee. Blood collection from human donors was approved under the ethical protocol \# NL5791207516.

\subsection{Materials}

Non-animal CM-chitosan was obtained by controlled derivatization as described [7-10] with proprietary modification [14]. A minimum $100 \mathrm{~g}$ of chitosan extracted from Agaricus bisporus were dispersed in a reaction medium that was composed of isopropanol and sodium hydroxide. Monochloracetic acid (MCA) reacted with chitosan in suspension. The carboxymethyl derivative was subsequently acetylated with acetic anhydride. The resulting soluble polymer was then extensively purified by precipitation in ethanol. The precipitate was dried out to remove excess solvent and residual water. KiOmedine ${ }^{\circledR} \mathrm{CM}$-chitosan was formulated at $1.8-2.2 \%(\mathrm{w}: \mathrm{w})$ in phosphate-buffered water for injection at $\mathrm{pH} 7.2 \pm 0.2$ supplemented with $3.5 \%$ sorbitol (w:w). The biomaterial was filled in Type I glass syringes (BD Medical, France) and steam sterilized. All of the raw materials and excipients conformed to European Pharmacopoeia standards for injectable use.

\subsection{Evaluation of Biocompatibility}

\subsubsection{In-Vitro Cytotoxicity}

Cytotoxicity of KiOmedine ${ }^{\circledR}$ CM-chitosan was tested in vitro in direct contact at $50 \%, 25 \%, 12.5 \%$, and $6.25 \%$ dilutions (v:v) in EMEM10 on triplicate monolayers of L-929 mouse fibroblast cells. A negative control, a control blank, and a positive control were added as required. After dilution with the sample or controls, cells were incubated at $37 \pm 1{ }^{\circ} \mathrm{C}$ in the presence of $5 \pm 1 \% \mathrm{CO}_{2}$ for 24-26 hours. Following incubation, $20 \mu \mathrm{L}$ of MTS-PMS solution dispensed in each well were incubated during 120-135 minutes at $37 \pm 1{ }^{\circ} \mathrm{C}$ (humidified) in $5 \pm 1 \% \mathrm{CO}_{2}$. The percent viability for the test article was determined from the control blank by measuring the amount of brown formazan by the optical density at 492 $\mathrm{nm}$.

\subsubsection{In Vitro Genotoxicity}

Genotoxicity of KiOmedine ${ }^{\circledR}$ CM-chitosan was tested in vitro in the Bacterial Reverse Mutation Assay as the ability to induce reverse mutations at the histidine locus of the Salmonella typhimurium tester strains TA98, TA100, TA1535, and TA1537 or at the tryptophan locus of the Escherichia coli tester strain WP2uvrA. Tubes containing molten top agar were inoculated in triplicate with culture from one of the five tester strains, along with the test solution tested at the dose of 100 $\mu \mathrm{L} /$ plate of the test article diluted with $0.9 \%$ Sodium Chloride (SC) at the doses $100 \%, 50 \%, 25 \%, 12.5 \%, 6.25 \%$, and $3.125 \%$ (v:v). Phosphate buffer or rat liver S9 Mixture for metabolic activation were used as negative or positive controls. The mean number of revertants for the test article plates was compared to the mean number of revertants of the appropriate negative control plates for each of the five tester strains.

Genotoxicity of KiOmedine ${ }^{\circledR} \mathrm{CM}$-chitosan was also tested in vitro in the Mouse Lymphoma Assay as the ability to induce mouse lymphoma forward gene mutation for 4 hours in the presence and absence of metabolic activation and for 24 hours in the absence of metabolic activation. The mouse lymphoma L5178Y/TK+/- cell line, heterozygous at the thymidine kinase (tk) locus, was used for this assay. This study was conducted by exposing the cells to the sample and appropriate negative and positive controls. The treatment was performed with $10 \%$, $5 \%, 2.5 \%, 1.25 \%$, and $0.63 \%(\mathrm{v}: \mathrm{v})$ of the test article for 4 hours in the absence and the presence of metabolic activation and for 24 hours in the absence of metabolic activation. 


\subsubsection{In Vitro Haemolysis}

Blood was obtained from human donors, pooled, diluted, and added in triplicate tubes with KiOmedine ${ }^{\circledR}$ CM-chitosan in calcium and magnesium-free phosphate buffered saline CMF-DPBS (Dulbecco). Control blanks, a negative control, and positive controls were prepared in the same manner as the test article. The tubes were then maintained for at least 3 hours at $37 \pm 2{ }^{\circ} \mathrm{C}$ with gentle periodic inversions at approximately 30 minute intervals. Following incubation, suspensions were mixed gently and centrifuged. The resulting supernatant was added to Drabkin's reagent. Absorbance was measured spectrophotometrically at a wavelength of $540 \mathrm{~nm}$.

\subsubsection{Human Whole Blood Assay}

Blood was collected from 8 donors in hirudin-coated tubes for measuring C5a secretion and in lithium heparin tubes for measuring IL- 8 and IL-1 $\beta$ secretion. Each blood sample was controlled for its cell phenotype distribution. $300 \mu \mathrm{L}$ of 3 -fold diluted (in RPMI) anticoagulated blood was added to the $350 \mu \mathrm{L}$ of the test article diluted to $2.9 \mathrm{mg} / \mathrm{mL}$ of the extract in RPMI in order to reach a final concentration of $1.5 \mathrm{mg} / \mathrm{mL}$ of KiOmedine ${ }^{\circledR}$ CM-chitosan. The mixture was incubated for 4 hours or 18 hours at $37^{\circ} \mathrm{C}$ and $5 \% \mathrm{CO}_{2}$, for C5a or IL-8 and IL- $1 \beta$ secretion testing, respectively. Appropriate controls were added, including saline as the negative control, $500 \mathrm{ng} / \mathrm{mL}$ LipoPolySaccharide (LPS) as the positive control for IL8 and IL1 $\beta$, and the crosslinked HA Hylan G-F 20 (Hylan) diluted at $0.6 \mathrm{mg} / \mathrm{mL}$ as the commercial reference. After incubation, the plasma was harvested by centrifugation and pro-inflammatory markers were quantified by ELISA according to the supplier's instructions (C5a, Biolegend; IL1 $\beta$ and IL8, Ebioscience). Each sample was tested in duplicate.

\subsubsection{Sensitization in Guinea Pigs}

Delayed dermal contact sensitization of KiOmedine ${ }^{\circledR}$ CM-chitosan was evaluated in Dunkin-Hartley guinea pigs (Charles River Laboratories, France). On distinct sites, the sample was injected intradermally (Induction I in association with Freund's Complete Adjuvant and Induction II) and applied topically (Induction II) to ten animals. $0.9 \%$ sodium chloride (SC) was similarly used as the negative control in five animals. Following a recovery period, all of the animals received a challenge patch and an intradermal injection of the test article and the negative control. All sites were scored for the presence of erythema and edema at 24, 48, and 72 hours.

\subsubsection{Material-derived Pyrogenicity}

Material-derived pyrogenicity was evaluated following the intravenous injection of KiOmedine ${ }^{\circledR} \mathrm{CM}$-chitosan in rabbits. The test article was extracted in $0.9 \%$ sodium chloride (SC) at $0.2 \mathrm{~g} / \mathrm{mL}$. A $10 \mathrm{~mL} / \mathrm{kg}$ dose of the appropriate test article extract was injected intravenously to 3 rabbits. Rectal temperature was measured every 30 minutes for 3 hours after injection.

Bacterial endotoxins were preliminarily controlled on the sterile biomaterial using Limulus Amebocyte Lysate in accordance with compendial requirements.

\subsubsection{Twenty Six-week Subcutaneous Toxicity in Rats}

Chronic systemic toxicity of $\mathrm{KiOmedine} \AA \mathrm{CM}$-chitosan was evaluated in Sprague-Dawley rats (Charles River Laboratories, France) throughout a 26 week follow-up period after repeated subcutaneous injections of approximately $5 \mathrm{~mL} / \mathrm{kg}$ every four weeks. Thirty male and thirty female rats were randomly assigned to either $\mathrm{KiOmedine}{ }^{\circledR} \mathrm{CM}$-chitosan or saline $(0.9 \%$ sodium chloride) as the negative control (15/sex/group). Samples were injected subcutaneously at one site every four weeks at days $1,29,57,85,113,141$, and 169, corresponding to weighed-adjusted volumes of $2.0 \mathrm{~mL}$ in males and $1.4 \mathrm{~mL}$ in females on average. Rats were observed and weighed on a daily basis for overt signs of toxicity for a period of one week and were then weighed on a weekly basis for a period of up to 13 weeks. They were then weighed once every two weeks thereafter and on the day of euthanasia. After 26 weeks, blood samples were collected for haematology and clinical chemistry analysis, and the rats were euthanized. A necropsy was conducted; selected organs were collected, namely heart, liver, pancreas, stomach, adrenals, thyroids, kidneys, skin, spleen, thymus, lymph nodes, muscle, brain, testes/ovaries, lungs, bronchi, sternum (bone marrow), weighed, and they were examined macroscopically. A microscopic evaluation of the collected organs was conducted by a board-certified pathologist. The injection test sites were excised and examined macroscopically.

\subsubsection{Twelve-week Intraarticular Implantation in Rabbits}

Intraarticular (IA) injections of KiOmedine $(\mathbb{R})$ CM-chitosan (approximately $400 \mu \mathrm{L} /$ joint) were performed in New Zealand white rabbits (Charles River Laboratories, France) and local tissue effects were evaluated at 1, 4, and 12 weeks in comparison to a commercial crosslinked HA, Hylan G-F 20 (Genzyme Biosurgery), and saline ( $\mathrm{n}=10$ sites per article and per time period). At 1 week and 4 weeks, the test article and control articles were also compared to a negative control (saline solution). For the 12 week period, rabbits had two other injections performed at 4 week intervals. Following injections, detailed clinical examinations (swelling, heat, redness, and articular pain) were conducted on a daily basis for 7 days after the injection and then twice weekly. Upon necropsy, the synovial fluid smears were collected and stained with May Grünwald Giemsa (MGG). The knee joints were dissected for subsequent histological evaluation by a board-certified pathologist according to OARSI recommendations for this model [15]. After complete fixation in $10 \% \mathrm{NBF}$, synovial membranes, decalcified menisci, decalcified distal femurs, and tibias and cruciate ligaments were dehydrated in alcohol solutions of increasing concentrations, cleared in xylene and embedded paraffin. Sections were cut with a microtome (4.5 $\mu \mathrm{m}$ thickness) and stained with Haematoxylin-Eosin-Safranin (HES) or Movat Pentachrome (MP). In addition, decalcified distal femurs and tibias were stained with toluidine blue.

\subsubsection{Twelve-week Intradermal Implantation in Rabbits}

Intradermal (ID) injections of KiOmedine ${ }^{\circledR} \mathrm{CM}$-chitosan (approximately $200 \mu \mathrm{L} /$ site) were performed in New Zealand white rabbits (Charles River Laboratories, France) and local tissue effects were evaluated at 1, 4, and 12 weeks in 
comparison to a commercial linear HA, Belotero ${ }^{\circledR}$ Hydro (Merz Aesthetics), and saline ( $\mathrm{n}=12$ sites per article and per time period). Following injections, detailed clinical examinations were conducted on a daily basis for 7 days after the injection and then twice weekly. The Primary Irritation Index was defined by grading erythema and edema. Upon necropsy, injected sites were collected for histopathological evaluation by a board-certified pathologist. After complete fixation in $10 \% \mathrm{NBF}$ for 48 hours, injected sites were dehydrated in alcohol solution, cleared in xylene, and embedded in paraffin. Central transverse sections were cut with a microtome at $4.5 \mu \mathrm{m}$ thickness and stained with HES.

\subsubsection{4-Day Immunotoxicity in Subcutaneous Air Pouch Mice}

Subcutaneous (SC) injections of KiOmedine $\AA$ CM-chitosan (approximately $1 \mathrm{~mL} /$ pouch) were performed in air pouch CD-1 mice (Janvier Labs) and local tissue effects were evaluated at 1, 3, 7, and 14 days. Saline and Hylan were used as the controls at day $1(\mathrm{n}=5$ animals per product) and day 14 ( $\mathrm{n}=10$ animals per product), and 1\% carrageenan was used as the control at day $1(n=5$ animals). Prior to injection, air pouches were induced by dorsal subcutaneous injection of $5 \mathrm{~mL}$ and $3 \mathrm{~mL}$ sterile air at day -7 and day -3 respectively. Animals were assessed for clinical signs, body weight, body temperature, blood biology (urea, creatinine, glycemia, alkaline phosphatase, and albumin), gross anatomy, weight of the organs, air pouch fluid, and skin histopathology at the various time points. Air pouch fluids were collected using 1.5 $\mathrm{ml}$ of $0.9 \%$ of $\mathrm{NaCl}$ injected into the pouch. The total lavage volume of exudate was recovered and weighed. The lavage volume of exudate was used for subsequent cell counting. The quantitation of proinflammatory markers TNF- $\alpha$, IL- $1 \beta$ and $\mathrm{KC} / \mathrm{CXCL1}$ were performed by ELISA according to the supplier's instructions (Abcam).

\subsection{Statistical Analyses}

Graphical representations and statistical analyses were performed using GraphPad Prism Software. The results are represented as mean \pm standard deviation. Histological scores were analysed by one-way ANOVA with Bonferroni's
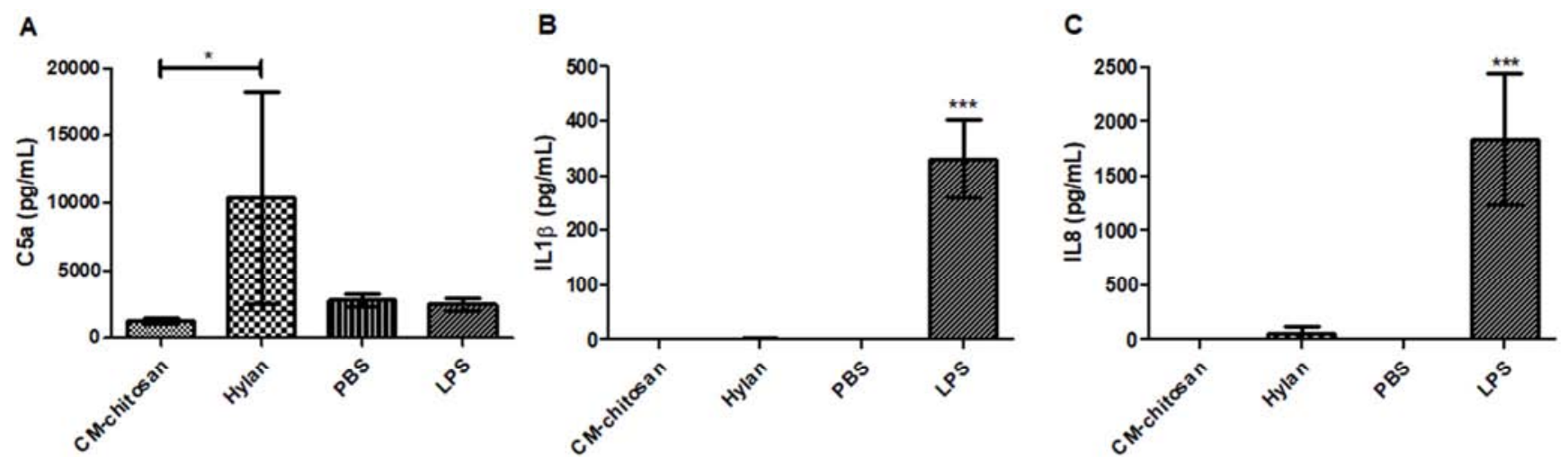

Figure 1. Activation of C5a (A), IL1 $\beta$ (B) and IL8 (C) after incubation with KiOmedine ${ }^{\circledR}$ CM-chitosan in human whole blood ( $n=8$ donors, mean $\pm 95 \%$ confidence interval, $* p<0.05 ; * * p<0.0001)$. 


\subsection{Material-derived Pyrogenicity}

No evidence of pyrogenicity was reported in rabbits. The sterile KiOmedine ${ }^{\circledR}$ CM-chitosan biomaterial was also tested for the absence of bacterial endotoxins that were found below the detection limit of $0.1 \mathrm{EU} / \mathrm{mL}$.

\subsection{Sensitization in Guinea Pigs}

Delayed sensitization of $\mathrm{KiOmedine}{ }^{\circledR} \mathrm{CM}$-chitosan was evaluated in the guinea pigs after topical and intradermal induction and challenge. The intradermal challenge was added to the topical challenge given the high molecular weight of $\mathrm{KiOmedine}{ }^{\circledR} \mathrm{CM}$-chitosan that would not migrate across the stratum corneu m. KiOmedine ${ }^{\circledR} \mathrm{CM}$-chitosan was considered to be non-irritant at a concentration of $100 \%$. After intradermal challenge, all guinea pigs were observed for immediate adverse reactions; local reactions and common clinical signs were considered unremarkable. No evidence of delayed sensitizing effects was observed by either topical challenge or intradermal challenge over the next 3 days.

\subsection{Chronic Systemic Toxicity in Rats}

Chronic systemic toxicity of $\mathrm{KiOmedine}{ }^{\circledR}$ CM-chitosan was evaluated in the rat model after repeated subcutaneous injections at approximately $5 \mathrm{~mL} / \mathrm{kg}$ bw every four weeks. No mortality was noted and all animals remained healthy during the study. There were no relevant peri-injection observations in both CM-chitosan and saline groups reported immediately after each subcutaneous injection and throughout the 26-week follow-up period. Furthermore, there were no relevant clinical signs or changes in body weight, in haematology or clinical chemistry parameters throughout the 26-week observation period in any of the rats. Upon necropsy, there were only a few macroscopic observations and their nature and distribution among both CM-chitosan and saline groups did not suggest any relationship to KiOmedine ${ }^{\circledR} \mathrm{CM}$-chitosan. In addition, there were no changes in organ weights. Microscopic evaluation of collected organs revealed no evidence of a systemic response to $\mathrm{KiOmedine}{ }^{\circledR} \mathrm{CM}$-chitosan. No significant change in haematology or clinical chemistry values could be attributed to KiOmedine ${ }^{\circledR}$ CM-chitosan

\subsection{Local Joint Effects in Rabbits}

The local tissue effects and degradation of KiOmedine ${ }^{\circledR}$ CM-chitosan was evaluated at 1, 4, and 12 weeks in comparison to Hylan and saline following intraarticular (IA) injection in rabbits. No mortality was noted and all of the animals remained healthy during the study. No relevant macroscopic observations, such as swelling or articular pain, were made at the injected sites either at baseline or during follow-up. In the KiOmedine ${ }^{\circledR}$ CM-chitosan group, no adverse local tissue effects were detected histopathologically in any tissue (synovial membranes, menisci, cruciate ligaments, tibial and femoral bones, including the articular cartilage) at week 1,4 , and 12 . Specifically, there were neither any signs of cytotoxicity nor adverse changes in the structure of the articular cartilage, chondrocyte density, or cluster formation at any time periods. No relevant cellular or tissue change was observed that was indicative of adverse synovial inflammation with $\mathrm{KiOmedine}{ }^{\circledR} \mathrm{CM}$-chitosan, except for a slight increase in synoviocytes and monocytes at week 1 (Figure 2). Polymorphonuclear cells were not significantly increased compared to saline, except at 4 weeks in the Hylan group (A); slight yet significant macrophage infiltration was observed at week 1 for both CM-chitosan and Hylan, and at week 4 and week 12 in the Hylan group only (B); giant cell formation was observed only in the Hylan group (C); significant proliferation and hypertrophy of synoviocytes was observed at week 1 in the CM-chitosan group and at week 12 in the Hylan group (D, E); significant synovial hyperplasia was observed at week 12 in the Hylan group (F). Tissue changes observed at week 4 and 12 in the Hylan group were indicative of synovial inflammation.

At week 1, the synovial smears from CM-chitosan and Hylan showed increased cellularity that was mostly composed of monocytes and synoviocytes (Figure 3 ). Cellularity mainly mostly consisted in vacuolated cells of the macrophagic lineage present in the synovial fluid and subsynovial space of the CM-chitosan group only, and it was considered to be consistent with intracellular storage of KiOmedine ${ }^{\circledR}$ CM-chitosan. Large numbers of synoviocytes and monocytes had intracytoplasmic microvacuoles (less frequent macrovacuoles) containing golden-brown, slightly refringent material (B). This KiOmedine ${ }^{\circledR}$ CM-chitosan storage was frequently associated with slight hypertrophy and hyperplasia of the synovial cells. KiOmedine ${ }^{\circledR} \mathrm{CM}$-chitosan residues were rarely detected at week 4 , which indicates its almost complete biodegradation. Twelve weeks after the first injection and subsequent injections repeated at 4 and 8 weeks, no relevant macroscopic observation was made for the KiOmedine ${ }^{\circledR}$ CM-chitosan group. KiOmedine ${ }^{\circledR}$ CM-chitosan residues were detected with overall comparable incidence and severity compared to the 4 week point. No adverse synovial inflammation or foreign body granuloma were observed (Figure 4). This suggested a lack of cumulative effects and proper degradation following repeated KiOmedine $\AA$ CM-chitosan injections.

In the Hylan group, adverse local tissue effects were present at week 4 and week 12 with increased cellularity, and they consisted of moderate granulomatous synovitis associated with a slight formation of lymphoid follicles, a slight to moderate villous hyperplasia of the synovial membrane, marked inflammatory changes in the synovial fluid, and granuloma formation at week 12 (Figure 4). In this group, synovitis was associated in one of these two sites with slight focal degenerative changes of the tibial cartilage. Globular components of the Hylan group had extracellular storage, mainly under the synovial membrane. At week 4 and week 12, the Hylan group did not show any relevant differences in terms of degradation compared to week 1. Adverse local tissue effects were increased in severity at week 12 when compared with week 4 . This suggested a slight cumulative effect of the repeated Hylan injections. One medial meniscus showed signs of erosion of the cartilage in the Hylan group. 
A

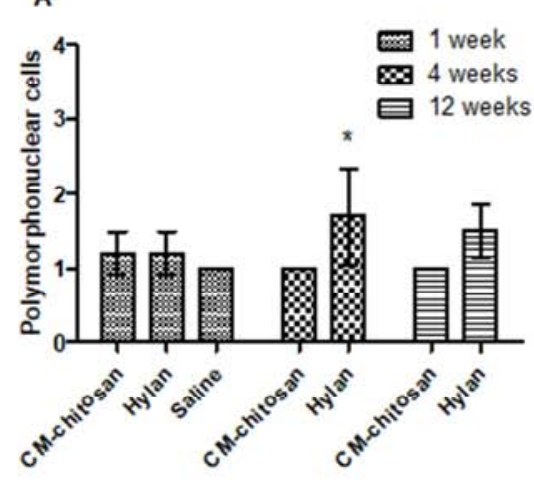

D

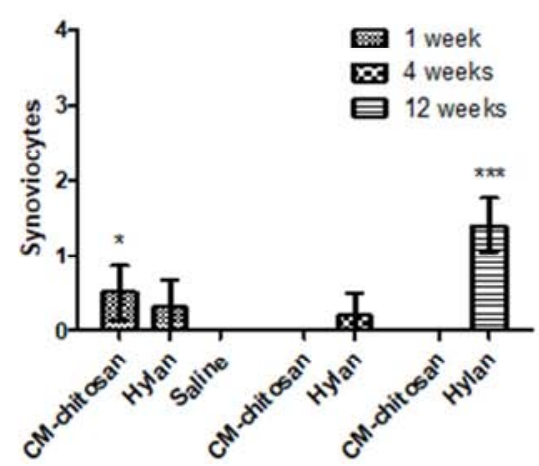

B

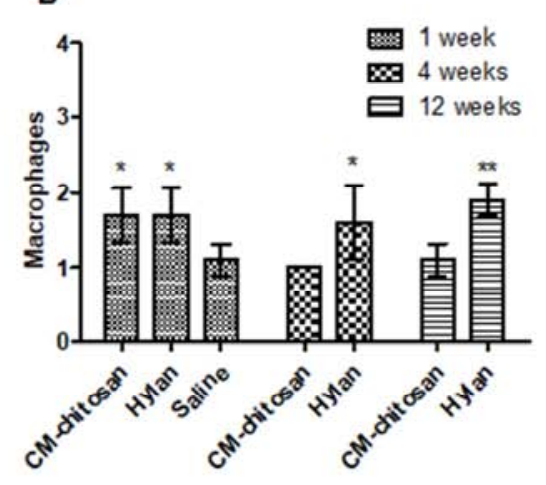

E

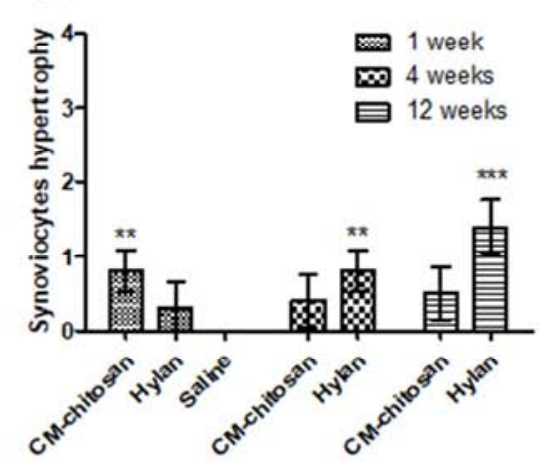

C

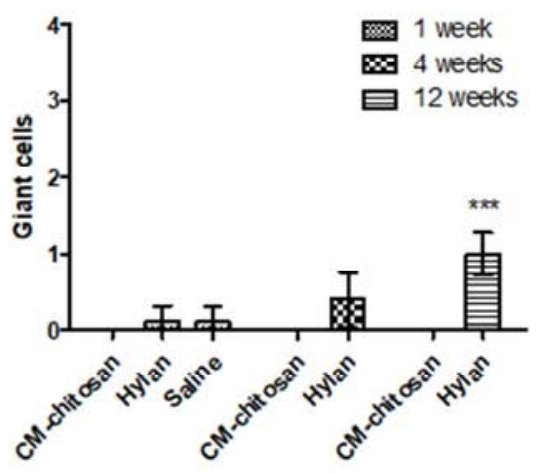

$F$

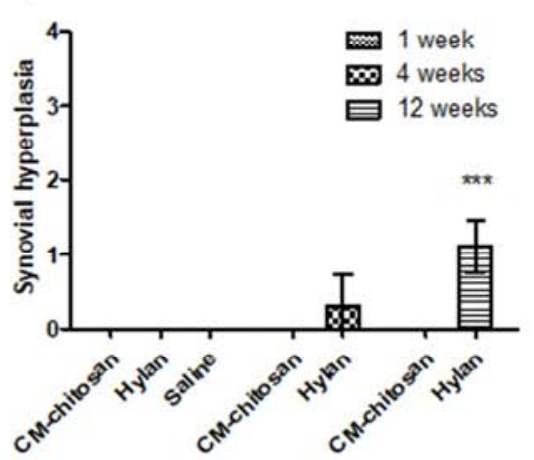

Figure 2. Histopathological evaluation of the cellularity and tissue change of the synovial membranes following IA injection of KiOmedine ${ }^{\circledR}$ CM-chitosan, Hylan or saline in rabbits $(400 \mu L / j o i n t, n=10$ sites per condition, mean $\pm 95 \%$ confidence interval, $* p<0.05 ; * * p<0.01 ; * * * p<0.0001)$.

A

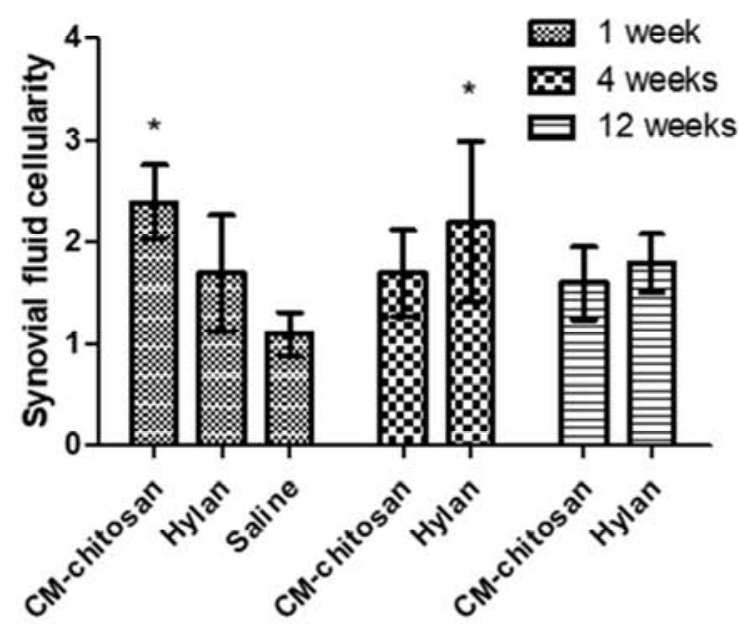

B

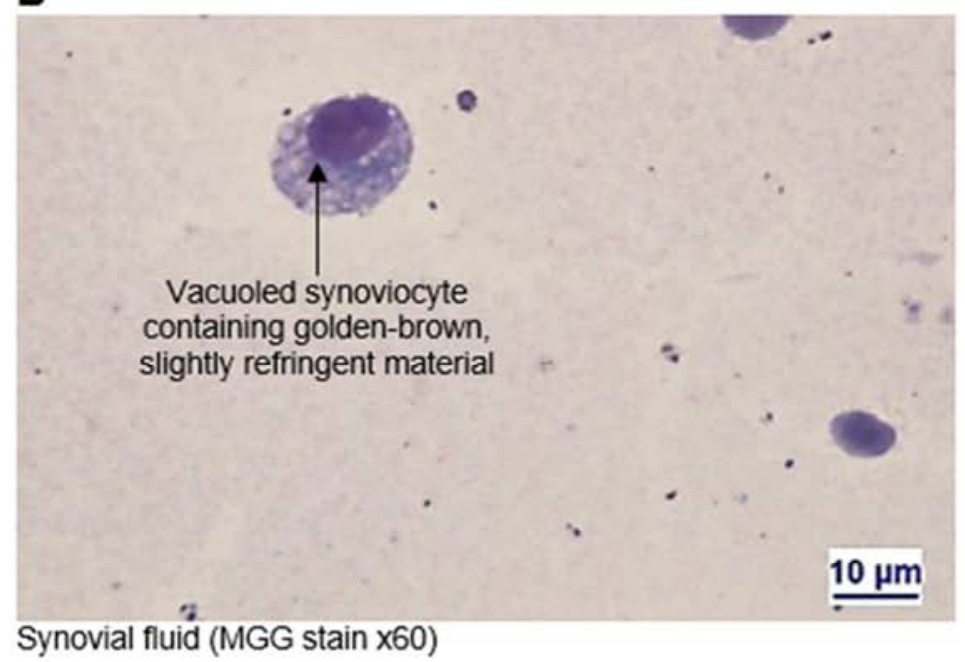

Figure 3. Cellularity of the synovial fluids (A) following IA injection of KiOmedine ${ }^{\circledR}$ CM-chitosan, Hylan or saline in rabbits (400 $\mu L / j o i n t, n=10$ sites per article and time period, mean $\pm 95 \%$ confidence interval, $\left.{ }^{*} p<0.05\right)$, and representative photomicrographs of CM-chitosan $(B)$.

In this IA injection study in the rabbit model, the synovial reaction to KiOmedine ${ }^{\circledR} \mathrm{CM}$-chitosan was graded as null to minimal at week 1, 4 and 12 when compared with saline or Hylan. No adverse local tissue effects in other joint compartments (menisci, cruciate ligaments, tibial and femoral bones including the articular cartilage) were observed for KiOmedine ${ }^{\circledR}$ CM-chitosan. The KiOmedine ${ }^{\circledR}$ CM-chitosan biomaterial demonstrated almost full degradation at 4 weeks, and alsoat 12 weeks following repeated injections at weeks 4 and 8. A lack of cumulative effects was confirmed. 
A

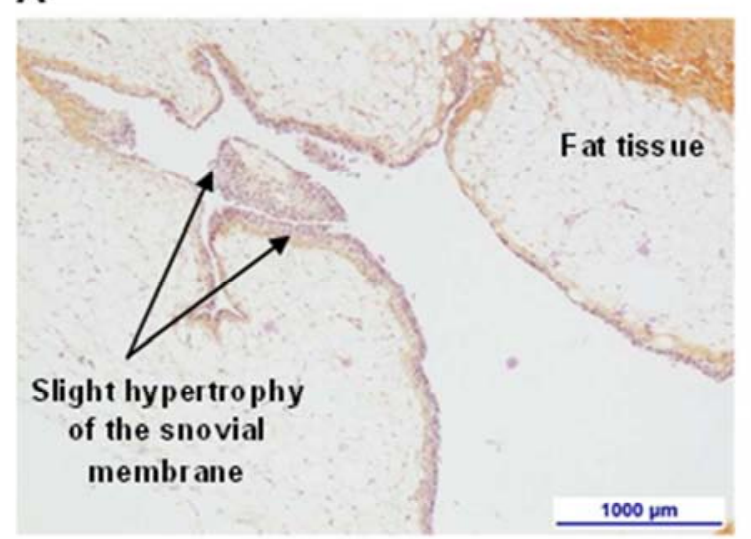

C

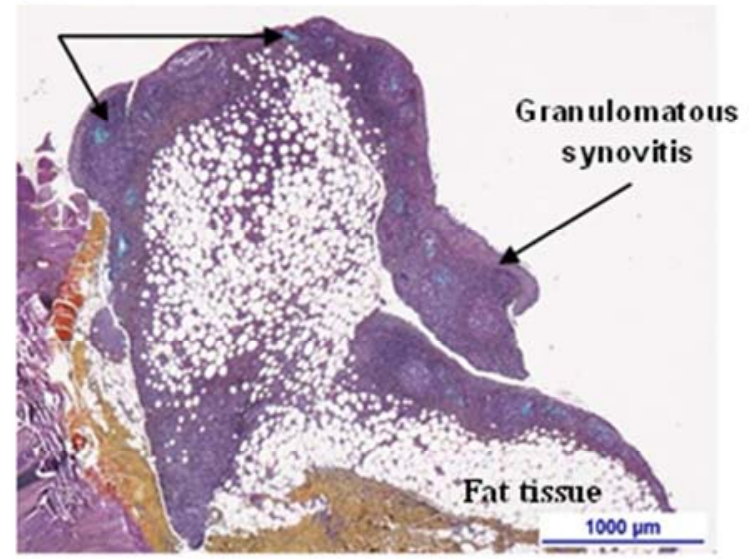

B

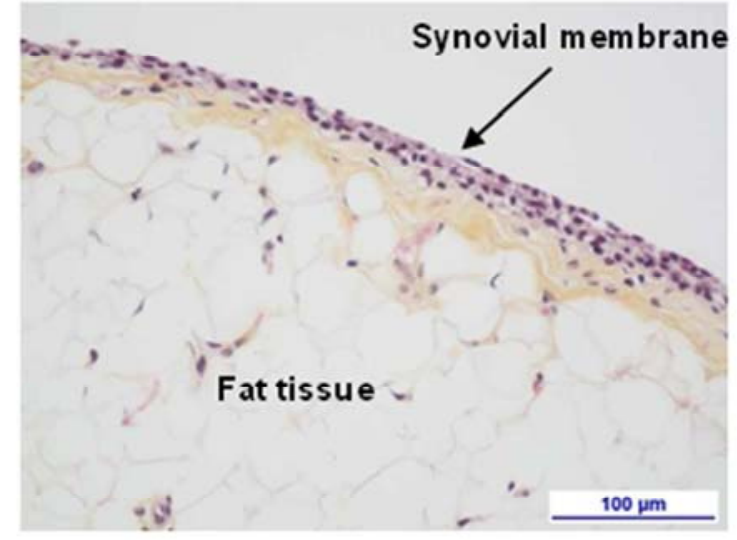

D

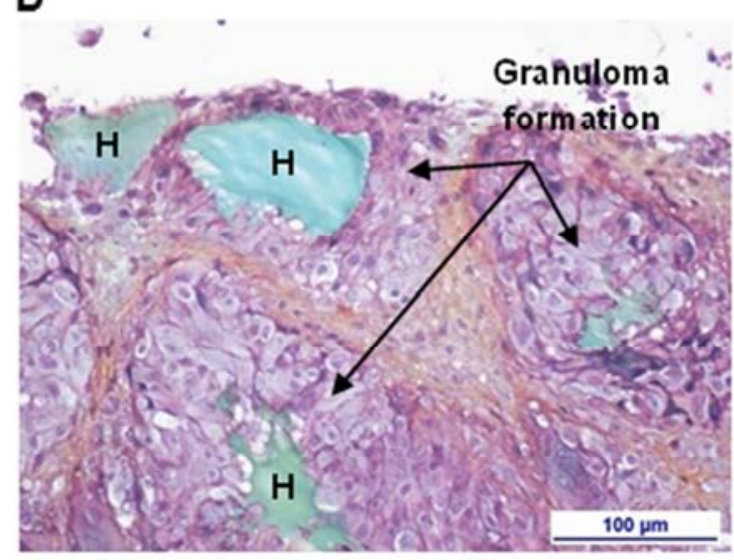

Figure 4. Representative photomicrographs of the synovial reaction after IA injection of KiOmedine ${ }^{\circledR} C M$-chitosan $(A, B)$ and Hylan $(C, D)$ at week 4 and at week 12 respectively.

\subsection{Local Dermal Effects in Rabbits}

The local tissue effects, irritation and degradation of KiOmedine ${ }^{\circledR}$ CM-chitosan was evaluated at 1,4 , and 12 weeks in comparison to linear HA and saline following intradermal (ID) injections in rabbits. No mortality was noted and all of the animals remained healthy during the study. No adverse local tissue reaction was macroscopically observed throughout the study after ID injection of CM-chitosan or linear HA when compared to the saline group, except very slight erythema from day 2 to day 5 in both groups (Figure 5).

Histopathologically, KiOmedine ${ }^{\circledR}$ CM-chitosan elicited very mild local tissue effects in the deep dermis at 1,4 , and 12 weeks, with no microscopic evidence of cytotoxicity. Slight fibroplasia was present at week 1 only. At 1 and 4 weeks, vacuolated macrophages were observed in the deep dermis and considered to represent either intracellular residues of KiOmedine ${ }^{\circledR}$ CM-chitosan or signs of macrophagic activation (Figure 6). Compared to saline, slight to moderate vacuolated or granular macrophages were observed in the deep dermis at week 1 and week 4 in both KiOmedine ${ }^{\circledR}$ CM-chitosan and linear HA groups, and was considered to represent either intracellular residues of material or signs of macrophagic activation (A). In the KiOmedine ${ }^{\circledR} \mathrm{CM}$-chitosan group, the tissue reaction was very minimal at week 4 and showed marked degradation of injected material and no fibroplasia (B); at week 12 , there was no adverse inflammation or foreign body reaction detected and the injected material had complete degradation (C). The degradation was marked at 4 weeks and complete by 12 weeks based on the presence of macrophages.

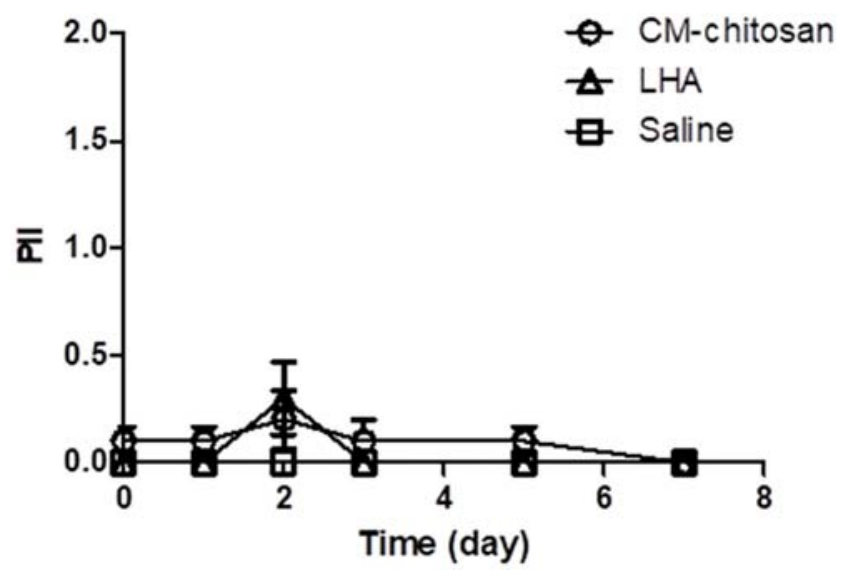

Figure 5. Primary Irritation Index (PII) of dermal tissues following the ID injection of KiOmedine ${ }^{\circledR}$ CM-chitosan, a linear HA (LHA) or saline in rabbits $(200 \mu \mathrm{L} /$ site, $n=12$ sites per article and time period, mean $\pm 95 \%$ confidence interval). PII values were calculated from erythema and edema scored 0 (none) to 4 (severe). 
A

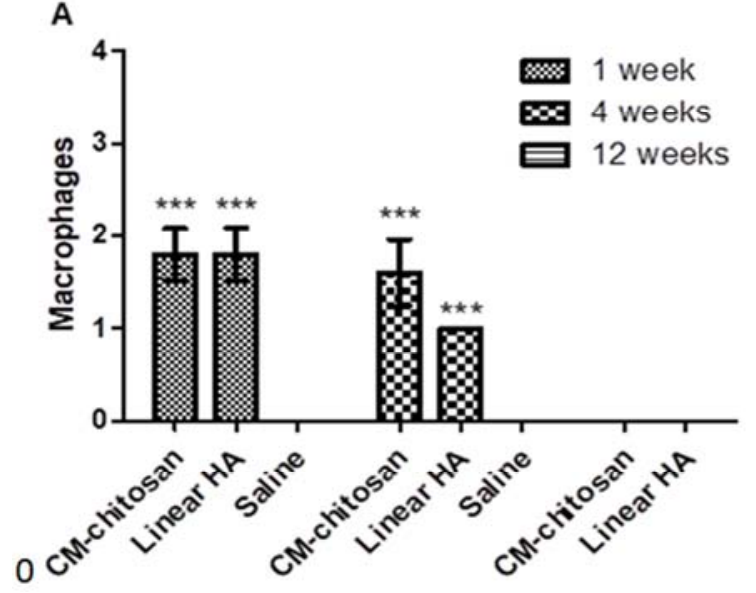

B

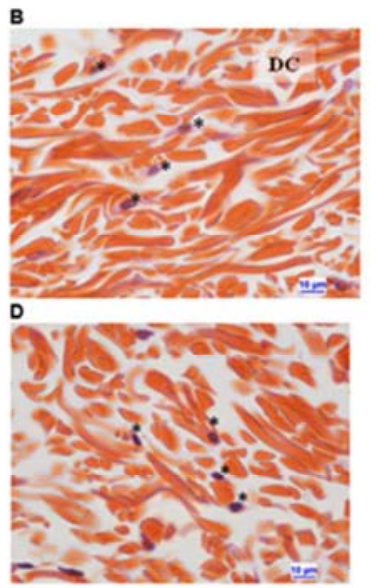

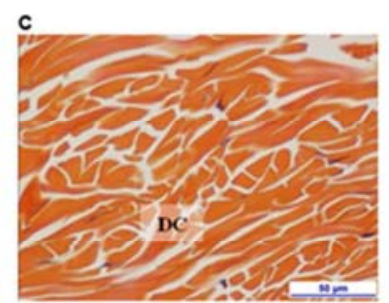

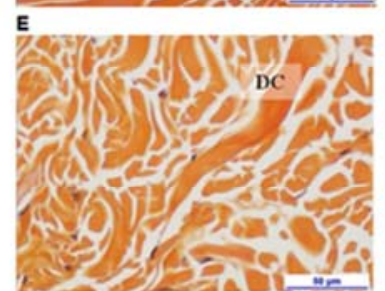

Figure 6. Macrophages infiltration of dermal tissues following the ID injection of KiOmedine ${ }^{\circledR} C M$-chitosan, a linear HA or saline in rabbits (200 $\mu L /$ site, $n=10$ sites per article and time period, mean $\pm 95 \%$ confidence interval, ***p $<0.0001)$, and representative photomicrographs of CM-chitosan (B, C) and linear HA (D, E) respectively at week 4 and at week 12 (DC: dermal collagen; *: macrophages filled with intracellular material).

Similar results in terms of local tissue effects and degradation were observed with linear HA. Microscopically, slight fibroplasia, as defined by increased numbers of fibroblasts and/or activated appearance of fibroblasts, was present at 1 week (data not shown). Linear HA elicited very mild local tissue effects in the deep dermis at 4 and 12 weeks after injection (D, E). Granular macrophages accounted for intracellular storage of HA residues. The degradation was almost complete at 4 weeks and it was complete by 12 weeks.

In this ID injection study in the rabbit model, KiOmedine ${ }^{\circledR}$ $\mathrm{CM}$-chitosan was found to be minimally irritant within 5 days after injection. Overall, the histopathologic analysis showed very mild local tissue effects that were comparable or even lower than linear HA.
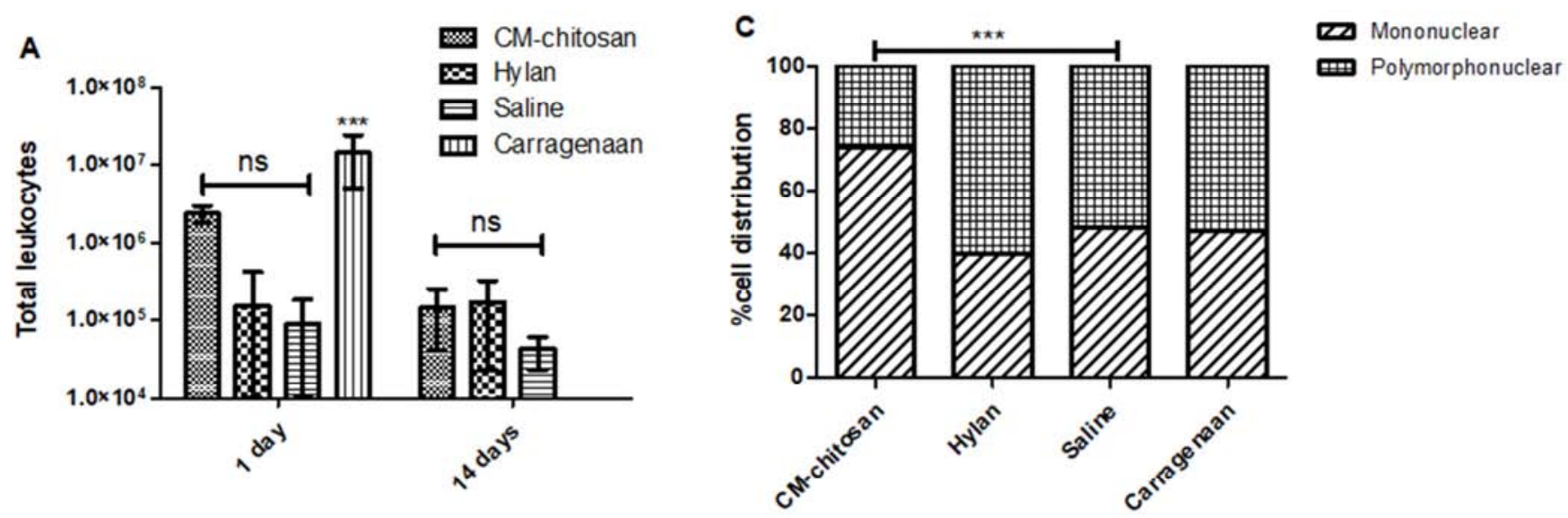

B

D
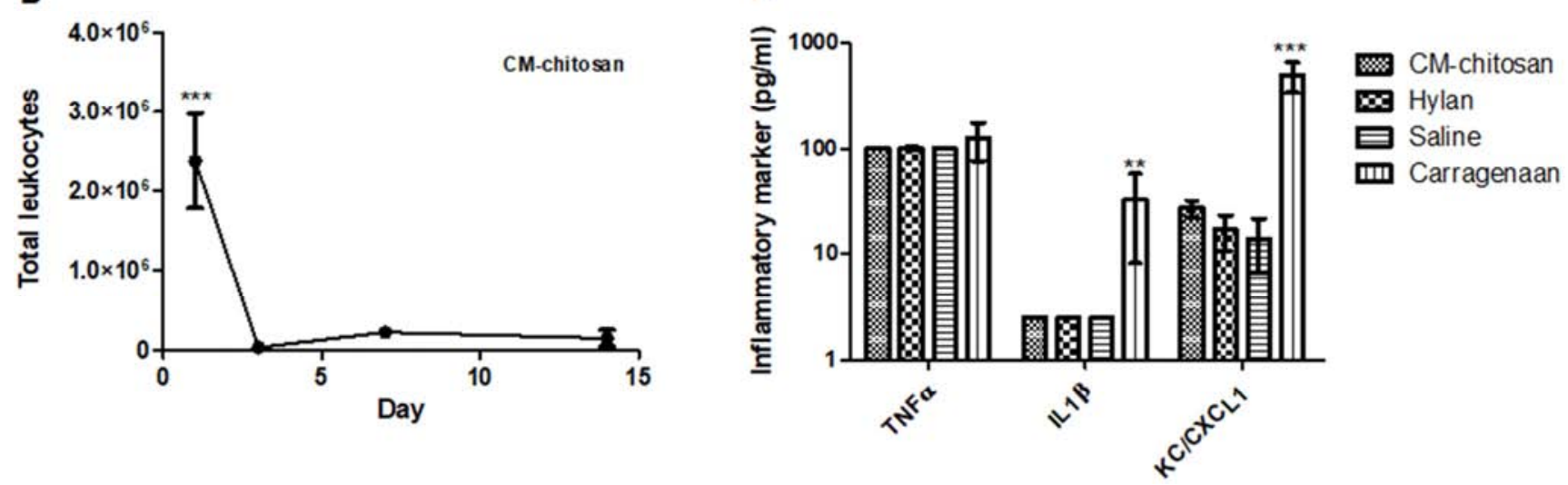

Figure 7. Evolution of the leukocyte formulation, cell distribution, and inflammatory markers (at day 1) following the subcutaneous injection of 1 mL KiOmedine ${ }^{\circledR}$ CM-chitosan in the mouse air pouch $(n=5$ sites at day 1 and 10 sites at day 14, mean $\pm 95 \%$ confidence interval, $* * p<0.01, * * * p<0.0001)$. 


\subsection{Immunotoxicity in Mouse Air Pouch}

Immunotoxicity was evaluated over 14 days after the subcutaneous injection of KiOmedine ${ }^{\circledR}$ CM-chitosan at approximately $28 \mathrm{~mL} / \mathrm{kg}$ bw in the mouse air pouch model. There were no changes indicative of any systemic toxicity in clinical observations, body weight, temperature, blood chemistry, organ weight, or macroscopic findings in major organs. Skin tissues around the pouch were found to be unremarkable for local effects by macroscopy and histology. There were no significant changes in macroscopy and cytology of the lavage fluids, except for a slight increase of mononuclear cells at day 1 , and either no or barely detectable stimulation of inflammation markers (IL-1 $\beta$, TNF- $\alpha$ and CXCL1) (Figure 7). Total leukocyte counts were not significantly different amongst the group at day 1 post-injection except in the group treated with carrageenan (A). In the KiOmedine ${ }^{\circledR} \mathrm{CM}$-chitosan group, a increase in the total leukocyte count was observed at day 1 post-injection which quickly returned to the baseline level at day 3 and onwards (B). At day 1 post-injection, the cellular proportion of mononuclear cells was increased in the CM-chitosan group as compared to the other groups (C). However, the levels of inflammatory markers remained undetectable orat their baseline values when compared to the positive control carrageenan (TNF- $\alpha$ detection limit of $100 \mathrm{pg} / \mathrm{mL}$; IL- $1 \beta$ detection limit of $2.5 \mathrm{pg} / \mathrm{mL}$; CXCL-1 detection limit of 8.75 $\mathrm{pg} / \mathrm{mL})(\mathrm{D})$.

\section{Discussion}

In this study, we present an innovative biomaterial KiOmedine ${ }^{\circledR}$ CM-Chitosan composed of non-animal CM-chitosan that was intended for use as an injectable bioabsorbable material for biomedical applications in regenerative medicine. Preliminary to its use in humans, extensive biocompatibility testing was conducted in order to support its biological safety evaluation.

Non-animal chitosan is derived from chitin extracted from the cell walls of the edible basidiomycete "white button" mushroom, A. bisporus. No significant toxicological concern was reported in the literature with food consumption of $A$. bisporus. In this study, KiOmedine ${ }^{\circledR}$ CM-chitosan extracted from $A$. bisporus showed no sign of cytotoxicity, genotoxicity, or haemolytic activity in vitro.

Allergenicity to $A$. bisporus is relatively rare. Few allergens were identified, a superoxide dismutase and a mannitol dehydrogenase [16] from the spores responsible for causing inhalative allergy; no allergen were identified causing ingestive allergy [17]. In this study, no sensitizing effect was observed by topical or intradermal challenges of KiOmedine ${ }^{\circledR}$ CM-chitosan in pre-immunized guinea pigs.

From literature review [18], there is no specific toxicological concern related to chitosan. To our knowledge, this study reports for the first time the systemic toxicity evaluation of CM-chitosan after repeated chronic exposure of approximately $100 \mathrm{mg} / \mathrm{kg}$ in the rat model. The 4 week timeframe between each injection was selected in order to uncover any toxic effects of degradation products related to KiOmedine ${ }^{\circledR}$ CM-chitosan. No organ-specific toxicity was observed upon macroscopy, blood chemistry, and histopathological evaluation. In Dong et al. [19, 20], absorption, distribution, metabolism, and excretion were evaluated in rat-injected intraperitoneally with $50 \mathrm{mg} / \mathrm{kg}$ of fluorescein isothiocyanate (FITC)-labelled CM-chitosan. More than $90 \%$ of FITC was rapidly absorbed from the abdominal cavity within 6 hours. Following absorption, FITC-labelled CM-chitosan was widely distributed into the blood system and most tissues. A rapid uptake was first noted in the liver and then a gradual increase was observed in the spleen and kidney after 3 days. CM-chitosan was still present in these organs $\sim 11$ days after intraperitoneal administration, while the content greatly decreased over time. Urinary extraction was found to be the major pathway for elimination of the FITC-labelled CM-chitosan. The distribution and excretion of CM-chitosan were accompanied with progressive reduction in molecular weight over time. Overall, the authors concluded favourably on the biodegradability and toxicity of CM-chitosan in vivo. These results were consistent with the biodegradation pathway of native chitosan [21] and HA [22]. In the present study, no organ-specific toxicity was reported, particularly at the level of the liver, spleen, and kidney, which are the main organs involved in the biodegradation of KiOmedine ${ }^{\circledR}$ CM-chitosan $[19,20]$.

Local effects and biodegradation of KiOmedine ${ }^{\circledR}$ $\mathrm{CM}$-chitosan were extensively evaluated in joint tissues and dermal tissues in the rabbit model. In these studies, no macroscopic observation was made suggesting adverse tissue irritation or inflammation following injection. In the IA rabbit study, KiOmedine ${ }^{\circledR}$ CM-chitosan did not induce any relevant synovial inflammation although a slight synovial reactivity was observed shortly after injection which seemed to be related to the biodegradation process of the material. No cytopathogenicity or deleterious consequences on the cartilage and other joint structures were noted. No cumulative effect was evidenced following repeated injections at 4 week intervals as opposed to the reference product Hylan G-F 20. Following IA injection in the rabbit model, the latter crosslinked HA was associated with marked inflammation of the synovial fluid, increased synovial reactivity, granuloma formation, and occasionally slight focal cartilage degeneration. IA injections of Hylan GF-20 have been associated with granulomatous reactions [23, 24], immune responses including hypersensitivity to avian proteins covalently bound to HA [25, 26], or possible complement activation [27] caused by the material itself.

KiOmedine ${ }^{\circledR}$ CM-chitosan was found to be equally well tolerated following intradermal injections in the rabbit model. Macroscopically, minimal erythema not more than grade 1 was occasionally observed at around day 3 ; the local reaction was self-limited and recovered within one week. Minimal irritation was also observed after the injection of linear HA. 
No histopathological evidence of adverse local effects, foreign body granulomas, or cytotoxicity was reported in the dermal tissues. Foreign body granuloma is another serious complication that is occasionally reported after the injections of crosslinked HA or other biomaterials such as polylactid [28, 29]. A granuloma is a chronic inflammatory reaction that is composed of a collection of immune cells, mainly macrophages. In the context of injectable biomaterials, a process of encapsulation occurs when the biomaterial exhibits improper biodegradation from the tissues and this leads to its encapsulation by immune cells. Macrophages are activated and fused into multinucleated giant cells. Histologically, palisaded granulomatous tissue mainly composed of macrophages and giant cells is usually discovered in a sterile abscess or nodule. The interaction between CM-chitosan and macrophages is also evidenced in the literature [30-32]. In our implantation studies, the biodegradation of KiOmedine ${ }^{\circledR}$ CM-chitosan from the injection site involved the recruitment of vacuolated cells of the macrophagic lineage in both joint and dermal tissues on week 1 post-injection. The cellular infiltrate was spontaneously resolved within approximately 4 weeks after the complete clearance of CM-chitosan from the injection site. No granuloma formation was observed in vivo with $\mathrm{KiOmedine}{ }^{\circledR} \mathrm{CM}$-chitosan during or after its complete degradation. Overall, the histopathologic analysis showed very mild local tissue effects that were comparable or even lower than linear HA.

Finally, the immunotoxicological profile of $\mathrm{KiOmedine}{ }^{\circledR}$ CM-chitosan was investigated in the mouse air pouch model. In this model, the air pouch is produced by the subcutaneous injection of sterile air into the back of a mouse or rat. The pouch generated by air inflation provides a localized environment in which to study cell trafficking and the inflammatory response [33, 34]. This model has been extensively used to evaluate the inflammatory response to biomaterials, including chitosan [35, 36] and Hylan [26, 37].
In the present study, no systemic reaction was reported in response to the high dose injection of KiOmedine ${ }^{\circledR}$ CM-chitosan at $\sim 560 \mathrm{mg} / \mathrm{g}$. Compared to the controls, the cytological analysis showed no significant change in cellularity associated with the treatment, except for a slight increase in mononuclear cells observed at day 1 which rapidly decreased to baseline at day 3 . Soluble markers representative of inflammatory responses to biomaterials, namely two proinflammatory cytokines IL- $\beta$ and TNF- $\alpha$, and the neutrophil-attracting chemokine $\mathrm{KC} / \mathrm{CXCL} 1$, were all unremarkable in that in vivo model. Furthermore, KiOmedine ${ }^{\circledR}$ CM-chitosan was not found to potentiate the activation of the central complement component C5a or any release of inflammatory mediator IL-1 $\beta$ and neutrophil-attracting chemokine IL-8 in human whole blood. Overall, it was concluded that KiOmedine ${ }^{\circledR}$ CM-chitosan had minimal potential to induce immunotoxic reactions.

\section{Conclusion}

In summary, our study represents an unprecedented body of work supporting the biological safety evaluation of an innovative biomaterial $\mathrm{KiOmedine}{ }^{\circledR} \mathrm{CM}$-chitosan composed of non-animal CM-chitosan for subsequent clinical use in injectable medical devices (Table 1). All biocompatibility test requirements were met in accordance with the ISO 10993 series. Overall, KiOmedine ${ }^{\circledR} \mathrm{CM}$-chitosan produced from $A$. bisporus is regarded as a safe material with no cytotoxicity, no genotoxicity, minimal toxic effects, and low biological effects on the targeted tissues. Its biodegradation process has been appropriately characterized and it is not associated with deleterious effects for the host tissues. The biological safety profile of $\mathrm{KiOmedine}{ }^{\circledR} \mathrm{CM}$-chitosan described in this study warrants clinical investigations of this biomaterial into humans.

Table 1. Biological safety summary of KiOmedine ${ }^{\circledR} C M$-chitosan.

\begin{tabular}{|c|c|c|}
\hline Biological safety & Test model & Results \\
\hline Cytotoxicity ISO10993-5 & In vitro mouse fibroblast & No cytotoxicity. \\
\hline Genotoxicity ISO10993-3 & $\begin{array}{l}\text { In vitro BRMA } \\
\text { and MLA }\end{array}$ & No genotoxicity. \\
\hline Haemocompatibility ISO10993-4 & $\begin{array}{l}\text { In vitro human red blood cell haemolysis } \\
\text { In vitro human whole blood assay }\end{array}$ & $\begin{array}{l}\text { No haemolysis }<2 \% \text {. } \\
\text { No blood activation for C } 5 \text { a, IL1 } \beta \text { and IL8. }\end{array}$ \\
\hline Pyrogenicity & Rabbit pyrogen test & No pyrogenicity in rabbits. \\
\hline Sensitization ISO10993-10 & Guinea pig maximization test & No sensitization following topical and intradermal application. \\
\hline Systemic toxicity ISO10993-11 & $\begin{array}{l}\text { 26-week toxicity after repeated } \\
\text { subcutaneous injections in rats }\end{array}$ & $\begin{array}{l}\text { No systemic toxicity at } 26 \text { weeks of } 7 \text { repeated injections of } \\
\text { CM-chitosan at } \sim 100 \mathrm{mg} / \mathrm{kg} \text { at } 4 \text { weeks interval. }\end{array}$ \\
\hline Local tissue effects ISO10993-6 & 12-week intraarticular injection in rabbits & $\begin{array}{l}\text { No macroscopic or microscopic sign of irritation and adverse local } \\
\text { effects on articular tissues at } 1 \text { week, } 4 \text { weeks, and } 12 \text { weeks after } 3 \\
\text { repeated intraarticular injections at } 4 \text { week intervals. } \\
\text { Minimal sign of dermal irritation. No microscopic sign of adverse local } \\
\text { effects on dermal tissues at } 1 \text { week, } 4 \text { weeks, and } 12 \text { weeks. }\end{array}$ \\
\hline Immunotoxicity ISO10993-20 & $\begin{array}{l}\text { 14-day immunotoxicity in the } \\
\text { subcutaneous mouse air pouch }\end{array}$ & $\begin{array}{l}\text { No systemic or local immunotoxicity at } 1,3,7 \text {, and } 14 \text { weeks of a } \\
\text { single dose injection of CM-chitosan at } \sim 560 \mathrm{mg} / \mathrm{kg} \text {. }\end{array}$ \\
\hline
\end{tabular}

\section{Acknowledgements}

Walloon Government and "Service public de Wallonie, Direction des Projets de Recherche" in the framework of

This work was supported by research funds from the project $\mathrm{n}^{\circ} 7842$ and project $\mathrm{n}^{\circ} 7360$. 


\section{References}

[1] Rodríguez-Vázquez $M$, Vega-Ruiz $B$, Ramos-Zúñiga $R$, Saldaña-Koppel DA, Quiñones-Olvera LF. Chitosan and Its Potential Use as a Scaffold for Tissue Engineering in Regenerative Medicine. Biomed Res Int. 2015; 2015: 821279.

[2] Cheung RC, Ng TB, Wong JH, Chan WY. Chitosan: An Update on Potential Biomedical and Pharmaceutical Applications. Mar Drugs. 2015 Aug 14; 13 (8): 5156-86.

[3] Kumar, MNV. A Review of Chitin and Chitosan Applications. Reactive and Functional Polymers, 2000, 46, 1-27.

[4] Kumirska J, Czerwicka M, Kaczyński Z, Bychowska A, Brzozowski K, Thöming J, Stepnowski P. Application of spectroscopic methods for structural analysis of chitin and chitosan. Mar Drugs. 2010 Apr 29; 8 (5): 1567-636.

[5] Trujillo. Preparation of carboxymethylchitin. Carbohydr. Res. 7 (1968) 483-485.

[6] Jimtaisong A, Saewan N. Utilization of carboxymethyl chitosan in cosmetics. Int J Cosm Sci, 2014, (36): 12-21.

[7] Chen X-G, Park H-J. Chemical characteristics of O-carboxymethyl chitosans related to the preparation conditions. Carbohydr Polymers. 2003, 53: 355-359.

[8] de Abreu FR, Campana-Filho SP. Preparation and characterization of carboxymethylchitosan. Polimeros. 2005, 15 (2): 79-83.

[9] de Abreu FR, Campana-Filho, S. P. Characteristics and properties of carboxymethylchitosan. Carbohydr Polymers. 2009, 75: 214-221.

[10] Bukzem AL, Signini R, Dos Santos DM, Lião LM, Ascheri DP. Optimization of carboxymethyl chitosan synthesis using response surface methodology and desirability function. Int J Biol Macromol. 2016 Apr; 85: 615-24.

[11] Bellich B, D'Agostino I, Semeraro S, Gamini A, Cesàro A. The Good, the Bad and the Ugly of Chitosans. Mar Drugs. 2016 May 17; 14 (5): 99.

[12] Ravindranathan S, Koppolu BP, Smith SG, Zaharoff DA. Effect of Chitosan Properties on Immunoreactivity. Mar Drugs. 2016 May $11 ; 14$ (5).

[13] Yu MM, Jiang TF, Wang YH, Wang DY, Li ZH. Identification and analysis of an impurity inducing clinical adverse effect in anti-adhesion carboxymethyl chitosan products. J Pharm Biomed Anal. 2013 Nov; 85: 21-7.

[14] Chausson M, Douette P, Gautier S, Vaesen P, Choumane H, Rocasalbas G. Carboxyalkyl chitosan. PCT/EP2018/080767.

[15] Laverty S, Girard CA, Williams JM, Hunziker EB, Pritzker KP. The OARSI histopathology initiative - recommendations for histological assessments of osteoarthritis in the rabbit. Osteoarthritis Cartilage. 2010 Oct; 18 Suppl 3: S53-65.

[16] Gabriel MF, González-Delgado P, Postigo I, Fernández J, Soriano V, Cueva B, Martínez J. From respiratory sensitization to food allergy: Anaphylactic reaction after ingestion of mushrooms (Agaricus bisporus). Med Mycol Case Rep. 2015 Feb 24; 8: 14-6.
[17] Ho MHK, Hill DJ. White button mushroom food hypersensitivity in a child. J. Paed. Child Health (2006) Volume 42, Issue 9, 555-556.

[18] Baldrick P. The safety of chitosan as a pharmaceutical excipient. ReG. Toxicol. Pharmacol. 2010, 56: 290-299.

[19] Dong W, et al. Pharmacokinetics and biodegradation mechanisms of a versatile carboxymethyl derivative of chitosan in rats: in vivo and in vitro evaluation. Biomacromolecules. 2010 Jun 14; 11 (6): 1527-33.

[20] Dong W, et al. Effects of molecular weights on the absorption, distribution and urinary excretion of intraperitoneally administrated carboxymethyl chitosan in rats. J Mater Sci Mater Med. 2012 Dec; 23 (12): 2945-52.

[21] Onishi H, Machida Y. Biodegradation and distribution of water-soluble chitosan in mice. Biomaterials 1999 (20): 175-182.

[22] Necas J, Bartosikova L., Brauner P, Kolar J. Hyaluronic acid (hyaluronan): a review. Veterinarni Medicina, 53, 2008 (8): 397-411.

[23] Chen AL, Desai P, Adler EM, Di Cesare PE. Granulomatous inflammation after Hylan G-F 20 viscosupplementation of the knee: a report of six cases. J Bone Joint Surg Am. 2002 Jul; 84-A (7): 1142-7.

[24] Sasaki M, Miyazaki Y, Takahashi T. Hylan G-F 20 induces delayed foreign body inflammation in Guinea pigs and rabbits. Toxicol Pathol. 2003 May-Jun; 31 (3): 321-5.

[25] Hamburger M, Settles M, Teutsch J. Identification of an immunogenic candidate for the elicitation of severe acute inflammatory reactions (SAIRs) to hylan G-F 20. Osteoarthritis Cartilage. 2005 Mar; 13 (3): 266-8.

[26] Ishikawa M, Yoshioka K, Urano K, Tanaka Y, Hatanaka T, Nii A. Biocompatibility of cross-linked hyaluronate (Gel-200) for the treatment of knee osteoarthritis. Osteoarthritis Cartilage. 2014 Nov; 22 (11): 1902-9.

[27] Dragomir CL, Scott JL, Perino G, Adler R, Fealy S, Goldring MB. Acute inflammation with induction of anaphylatoxin C5a and terminal complement complex C5b-9 associated with multiple intra-articular injections of hylan G-F 20: a case report. Osteoarthritis Cartilage. 2012 Jul; 20 (7): 791-5.

[28] Lee JM, Kim YJ. Foreign body granulomas after the use of dermal fillers: pathophysiology, clinical appearance, histologic features, and treatment. Arch Plast Surg. 2015 Mar; 42 (2): 232-9.

[29] Lemperle G, Gauthier-Hazan N, Wolters M, Eisemann-Klein M, Zimmermann U, Duffy DM. Foreign body granulomas after all injectable dermal fillers: part 1. Possible causes. Plast Reconstr Surg. 2009 Jun; 123 (6): 1842-63.

[30] Nishimura K, Nishimura S, Nishi N, Saiki I, Tokura S, Azuma I Immunological activity of chitin and its derivatives. Vaccine. 1984 Mar; 2 (1): 93-9.

[31] Nishimura S, Ikeuchi Y, Tokura S. The adsorption of bovine blood proteins onto the surface of O-(carboxymethyl) chitin. Carbohydr Res. 1984 Dec 1; 134 (2): 305-12.

[32] Tokura S, Tamura H, Azuma I. Immunological aspects of chitin and chitin derivatives administered to animals. EXS. 1999; 87: 279-92. 
[33] Edwards JC, Sedgwick AD, Willoughby DA. The formation of a structure with the features of synovial lining by subcutaneous injection of air: an in vivo tissue culture system. J Pathol. 1981; 134: $147-56$.

[34] Sedgwick AD, Sin YM, Edwards JC, Willoughby DA. Increased inflammatory reactivity in newly formed lining tissue. J Pathol. 1983; 141: 483-95.

[35] Barbosa JN, Amaral IF, Aguas AP, Barbosa MA. Evaluation of the effect of the degree of acetylation on the inflammatory response to $3 \mathrm{D}$ porous chitosan scaffolds. J Biomed Mater Res A. 2010 Apr; 93 (1): 20-8.
[36] Vasconcelos DP, Costa M, Amaral IF, Barbosa MA, Águas AP, Barbosa JN Modulation of the inflammatory response to chitosan through M2 macrophage polarization using pro-resolution mediators. Biomaterials. 2015 Jan; 37: 116-23.

[37] Wooley PH, Song Z, Harrison A. Hyaluronic acid viscosupplements from avian and non-mammalian sources exhibit biocompatibility profiles with unique, source-specific, antigenic profiles. J Biomed Mater Res B Appl Biomater. 2012 Apr; 100 (3): 808-16. 